\title{
When Isolated Horizons met Near Horizon Geometries
}

\author{
Jerzy Lewandowski * Adam Szereszewski * $^{\dagger}$ and Piotr Walukt \\ Instytut Fizyki Teoretycznej, Uniwersytet Warszawski, \\ ul. Pasteura 5, 02-093 Warsaw, Poland
}

\begin{abstract}
There are two mathematical relativity frameworks generalizing the black hole theory: the theory of isolated horizons (IH) and the theory of near horizon geometries (NHG). We outline here and discuss the derivation of the NHG from the theory of IH by composing spacetimes from IH. The simplest but still quite general class of solutions to Einstein's equations of this type defines spacetimes foliated by Killing horizons emanating from extremal horizons. That derivation, clearly being a link between the two frameworks, seems to be unknown to the NHG researchers and is hardly acknowledged in reviews on the IH. This lecture was a contribution to the Mathematical Structures session of the 2nd LeCosPA International Symposium "Everything about Gravity" celebrating the centenary of Einstein's General Relativity on December 14-18, 2015 in Taipei.
\end{abstract}

Keywords: Isolated horizon; Near horizon geometry; Kundt spacetime

\section{INTRODUCTION}

A family of solutions to 4d-Einstein's equations was constructed in [1] from the following data:

- $S$ - a 2 dimensional manifold diffeomorphic to a sphere,

- $g=g_{A B} d x^{A} d x^{B}$ - a metric tensor on $S$,

- $\omega=\omega_{A} d x^{A}-$ a differential 1-form on $S$,

such that the following (tensor) equation is satisfied:

$$
D_{(A} \omega_{B)}+\omega_{A} \omega_{B}-\frac{1}{2} R_{A B}=0,
$$

where $D_{A}$ and $R_{A B}$ are, respectively, the torsion free covariant derivative and the Ricci tensor, defined on $S$ by the metric $g_{A B}$.

The corresponding solution to the vacuum Einstein equations is a metric tensor $g_{\mu \nu}$ defined on

$$
S \times \mathbb{R} \times \mathbb{R}
$$

*Jerzy.Lewandowski@fuw.edu.pl

Adam.Szereszewski@fuw.edu.pl

tPiotr.Waluk@fuw.edu.pl 
in terms of coordinates

$$
\left(x^{\mu}\right)=\left(x^{A}, v, u\right)
$$

in the following way

$$
g_{\mu \nu} d x^{\mu} d x^{\nu}:=g_{A B} d x^{A} d x^{B}-2 d u\left(d v-2 v \omega-\frac{1}{2} v^{2}\left(D_{A} \omega^{A}+2 \omega^{A} \omega_{A}\right) d u\right)
$$

\section{FOLIATION BY KILLING HORIZONS}

What is special about each of the solutions (2) is that the corresponding spacetime is foliated by Killing horizons. They are the surfaces

$$
u=\text { const }
$$

of the topology $S \times \mathbb{R}$ and

$$
v=0 .
$$

The Killing vector corresponding to each value $u=u_{0}$ is

$$
K^{\left(u_{0}\right)}=v \partial_{v}-\left(u-u_{0}\right) \partial_{u} .
$$

This was exactly the aim of performing the construction in [1]: to compose a spacetime out of horizons.

An unexpected property, though, was the emergence of an extremal Killing horizon

$$
v=0
$$

of the Killing vector

$$
K=\partial_{u}
$$

All the horizons (3) foliating the spacetime emanate from that extremal horizon.

The 1-form $\omega_{A} d x^{A}$, considered on a spacial slice

$$
v=\text { const }
$$

of any of the horizons (3) , coincides with minus the rotation 1-form potential, namely

$$
\nabla_{A} K^{\left(u_{0}\right)}=-\omega_{A} K^{\left(u_{0}\right)} .
$$

Considered on each spacial slice

$$
u=\text { const }
$$


of the extremal horizon (6), on the other hand, the 1-form $\omega_{A} d x^{A}$ coincides exactly with its rotation 1-form potential, that is, the pullback of the spacetime covariant derivative $\nabla_{\mu} K$ to the slice is

$$
\nabla_{A} K=\omega_{A} K
$$

The equation (11) coincides with the vacuum constraint that has to be satisfied by every induced metric tensor $g_{A B}$ and rotation 1-form potential $\omega_{A}$ on an extremal Killing or, more generally, isolated horizon, at which the vacuum Einstein equations hold [2]. The equation was studied in [3] for compact 2d-surfaces. It was proven therein that the only axisymmetric solutions $(g, \omega)$ admitted by $S$ diffeomeomorphic to $S^{2}$ are those provided by the horizons of extremal Kerr solutions. This may be interpreted as a quasi-local version of the black hole uniqueness theorem. Those solutions correspond to the Horowitz limit [4] of the extremal Kerr spacetimes, also known as the Kundt class solutions of the Petrov type D [5]. The equation (1) was farther studied in [6, 7]. However, intriguingly enough, still very little is known about a generic, axially non-symmetric case. It is not even known whether there exists any non-axisymmetric solution to eq. (11) on $S$ diffeomorphic to 2 -sphere. The other compact $2 \mathrm{~d}-S$ cases are either excluded — the higher genus case — or trivialized by the equation (11): on $S$ diffeomorphic to 2-torus, the only solutions are flat $g$ and $\omega=0$. It might be possible, though, that inclusion of a non-zero cosmological constant in the calculations would loosen these restrictions somehow. More specifically, as the argument relies on the definiteness of $\omega_{A} \omega^{A}-\Lambda$. A negative cosmological constant yet strengthens that property, and even eliminates the 2 -torus case, allowing only the 2 -sphere. However, a positive cosmological constant destroys the positive definiteness and we have no argument against the higher genus.

Above, Einstein's vacuum equations can be replaced by the Einstein-Maxwell vacuum equations. The extremal horizon constraint (10) is then suitably generalized by the presence of the Maxwell field. The uniqueness theorem still holds for the resulting extremal horizon constraint equation, with the Kerr solutions replaced by the Kerr-Newman solution [3]. The suitable generalization of the metrics (2) is also available [1]. Those metrics still have the form

$$
g_{\mu \nu}(x, u, v) d x^{\mu} d x^{\nu}=g_{A B}(x) d x^{A} d x^{B}-2 d u\left(d v-2 v \omega_{A}(x) d x^{A}-\frac{1}{2} v^{2} H(x) d u\right),
$$

where the function $H(x)$ depends now on both $\omega_{A}(x)$ and the Maxwell field [1].

\section{NEAR HORIZON LIMIT}

Yet more generally, metric tensors of the form (10), with arbitrary $g_{A B}(x), \omega_{A}(x)$, and $H(x)$ are called near horizon geometries [8]. The vacuum Einstein equations imposed on 
such a metric are equivalent to (112). In the case with matter, the equation (1) is generalized to

$$
D_{(A} \omega_{B)}+\omega_{A} \omega_{B}-\frac{1}{2} R_{A B}=\frac{1}{2} \mathcal{R}_{A B},
$$

where the new term $\mathcal{R}_{A B}$ is the pullback of the spacetime Ricci tensor to a spacial slice of an extremal Killing horizon [10]. The corresponding change in (20) amounts to a suitable modification of the function $H(x)$ [8].

The class of metric tensors (10) was derived by a neat argument [9] from the general class of metric tensors admitting an extremal Killing horizon. Let us recall. Suppose a spacetime metric $g$ admits a Killing horizon. Locally, in the neighbourhood of the horizon, $g$ can be written as

$$
g=g_{A B}(v, x) d x^{A} d x^{B}-2 d u\left(d v-2 v \omega_{A}(v, x) d x^{A}+\left(v \kappa H_{0}(v, x)+\frac{1}{2} v^{2} H(v, x)\right) d u\right) .
$$

The Killing vector is

$$
K=\partial_{u}
$$

and the Killing horizon is the surface

$$
v=0 .
$$

To remove the ambiguity of $\kappa$, we demand that $\left.H_{0}\right|_{v=0} \equiv-1$. Apart from that, $g_{A B}, \omega_{A}$, $H_{0}$, and $H$ are a priori arbitrary (differentiable) functions of the variables $\left(v, x^{A}\right)$. Under such circumstances, the $\kappa$ in (12) is a constant which coincides with the surface gravity of the Killing vector $K$ at the horizon (this actually holds also for a wide class of non-vacuum metrics, satisfying an energy inequality $T_{\alpha \beta} K^{\alpha} K^{\beta} \geq 0$ at the horizon). The horizon is extremal if and only if

$$
\kappa=0
$$

Then, the metric (12) admits the (pointwise) limit

$$
\epsilon \rightarrow 0
$$

of the transformation

$$
f_{\epsilon}:\left(v, u, x^{A}\right) \mapsto\left(\epsilon v, \frac{1}{\epsilon} u, x^{A}\right) .
$$

This is a generalization of the Horowitz limit mentioned before. The result is

$$
\left.\lim _{\epsilon \rightarrow 0} f_{\epsilon}^{*} g=g_{A B}(0, x) d x^{A} d x^{B}-2 d u\left(d v-2 v \omega_{A}(0, x) d x^{A}+\frac{1}{2} v^{2} H(0, x)\right) d u\right) .
$$


As the limit of the transformation (13), the resulting metric is $f_{\epsilon}$ invariant, that is, in addition to the original Killing vector field

$$
K=\partial_{u}
$$

it also has a second Killing vector field

$$
K^{(0)}=v \partial_{v}-u \partial_{u}
$$

Notice, that the surface $v=0$ is a Killing horizon also for $K^{(0)}$. It is, however, not an extremal horizon with respect to this second Killing field.

Moreover, every surface $u=u_{0}$ is the Killing horizon of a Killing vector field

$$
K^{(0)}+u_{0} K
$$

\section{CONCLUSIONS, OUTLOOK}

Another generalization of (11, 2) is also possible. Consider a spacetime foliated by nonexpanding horizons. Let the foliation be defined by

$$
u=\text { const. }
$$

Then, a distinguished null vector field $\ell$ tangent to all the horizons is

$$
\ell^{\mu}=g^{\mu \nu} u_{, \nu}
$$

On every spacial section $S$ of a leaf

$$
u=u_{0}
$$

of the foliation, the rotation 1 -form potential $-\omega_{A}$ is defined by

$$
\nabla_{A} \ell=-\omega_{A} \ell
$$

Suppose the spacetime satisfies vacuum Einstein's equations. Then, a constraint equation implied by Einstein's equations is again(!)

$$
D_{(A} \omega_{B)}+\omega_{A} \omega_{B}-\frac{1}{2} R_{A B}=0
$$

(we use the same notation as in (1)). It has to be satisfied on every leaf of the foliation independently (given a leaf, the independence of choice of slice $S$ is automatic, and follows from the 0th low of non-expanding horizons). So, it takes the mathematical form of the extremal horizon constraint even though there is no visible extremal horizon in the spacetime (and perhaps none at all). This mystery is yet to be understood. 
Constructing the family of solutions (2) to Einstein's equations by composing isolated horizons not only was a link between the theories of isolated horizons and theories of near horizon geometries (surprisingly, the paper [1] is unknown in the living reviews of those theories [8, 11]), but it also still contains intriguing mysteries. Throughout the paper we were assuming that the spacetime dimension is 4 . Most of the reasoningsubsubsectionInv generalizes to higher dimensions [8 -10] and there is still potential for new results. A nonaxially symmetric solution to the equation (10) would produce a new solution to Einstein's equations. Understanding the mysterious emergence of the extremal horizon constraint in the context of the spacetimes composed from non-extremal isolated horizons could also prove interesting.

\section{Acknowledgments}

We would like to thank Maciej Dunajski for pointing out the reference [9]. This work was partially supported by the Polish National Science Centre grant No. 2015/17/B/ST2/02871.

[1] T. Pawłowski, J. Lewandowski, J. Jezierski, Spacetimes foliated by Killing horizons, Class. Quant. Grav. 21 (2004), 1237-1252, arXiv:gr-qc/0306107.

[2] A. Ashtekar, C. Beetle, J. Lewandowski, Geometry of Generic Isolated Horizon, Class. Quant. Grav. 19 (2002), 1195-1225, arXiv:gr-qc/0111067.

[3] J. Lewandowski, T. Pawłowski, Extremal Isolated Horizons: A Local Uniqueness Theorem, Class. Quant. Grav. 20 (2003), 587-606, arXiv:gr-qc/0208032.

[4] J. M. Bardeen, G. T. Horowitz, The Extreme Kerr throat geometry: A Vacuum analog of $A d S(2) \times S^{2}$, Phys. Rev. D 60 (1999), 104030, arXiv:hep-th/9905099.

[5] H. Stephani,D. Kramer,M. MacCallum,C. Hoenselaers, E. Herlt, Exact Solutions to Einsteins Field Equations, Cambridge University Press, 2003.

[6] J. Jezierski, On the existence of Kundt's metrics and degenerate (or extremal) Killing horizons, Class. Quant. Grav. 26 (2009), 035011, arXiv:0806.0518 [gr-qc].

[7] J. Jezierski, B. Kaminski, Towards uniqueness of degenerate axially symmetric Killing horizon, Gen. Relativ. Gravit. 45 (2016), 987-1004, arXiv:1206.5136 [gr-qc].

[8] H. K. Kunduri, J. Lucietti, Classification of Near-Horizon Geometries of Extremal Black Holes, Living Rev. Rel. 16 (2013), 8, http://www.livingreviews.org/lrr-2013-8, arXiv:abs/1306.2517.

[9] H. S. Reall, Higher dimensional black holes and supersymmetry, Phys. Rev. D 68 (2003), 024024, arXiv:hep-th/0211290.

[10] J. Lewandowski, T. Pawłowski, Quasi-local rotating black holes in higher dimension: geometry, Class. Quant. Grav. 22 (2005), 1573-1598, arXiv:gr-qc/0410146.

[11] A. Ashtekar, B. Krishnan, Isolated and Dynamical Horizons and Their Applications, Living Rev. Rel. 7 (2004), 10, http://www.livingreviews.org/lrr-2004-10, arXiv:gr-qc/0407042. 